\title{
Localization of FAK is related with colorectal carcinogenesis
}

\author{
TOSHIHIRO MURATA ${ }^{1}$, YOSHIO NAOMOTO ${ }^{1}$, TOMOKI YAMATSUJI ${ }^{1}$, TAKAOMI OKAWA ${ }^{1}$, \\ YASUHIRO SHIRAKAWA ${ }^{1}$, MEHMET GUNDUZ ${ }^{2}$, TETSUJI NOBUHISA ${ }^{1}$, MUNENORI TAKAOKA $^{1}$, \\ MEHMET SIRMALI $^{3}$, MOTOWO NAKAJIMA ${ }^{4}$, YUKO OHNO ${ }^{5}$ and NORIAKI TANAKA ${ }^{1}$
}

Departments of ${ }^{1}$ Gastroenterological Surgery, Transplant, and Surgical Oncology, ${ }^{2}$ Oral Pathology and Medicine,

Okayama University Graduate School of Medicine, Dentistry and Pharmaceutical Sciences, Okayama, Japan;

${ }^{3}$ Department of Thoracic Surgery, Suleyman Demirel University, Isparta, Turkey; ${ }^{4}$ New Business and Technology Transfer, Johnson \& Johnson, Tokyo; ${ }^{5}$ Department of Health Sciences, Graduate School of Medicine, Osaka University, Osaka, Japan

Received November 19, 2007; Accepted January 5, 2008

\begin{abstract}
Focal adhesion kinase (FAK) is an important mediator functioning between cells and the extracellular matrix and is closely related with the integrin-signaling pathway. FAK has been reported to be involved in the proliferation, differentiation and apoptosis of cells. To date, no report has demonstrated the involvement of FAK in the carcinogenesis of the digestive tract. Therefore, we examined colorectal, esophageal, pancreatic and mammary cancers for expression of FAK and Phospho (P)-FAK by immunohistochemistry. Strong expression of FAK in the cytoplasm was detected in all 4 tumor types and expressions of FAK and P-FAK increased as the degree of cell differentiation became higher in colorectal and esophageal carcinomas. Interestingly P-FAK expression was confined to the nuclei, which was an unexpected result. No previous report of such a finding has been published for gastrointestinal cancer. All four of the organs investigated in the present study showed P-FAK expression in the nuclei, suggesting an association between FAK activation and abnormal cell proliferation. We also performed immunostaining of P-FAK in cell lines to examine the significance of its experience in the nuclei. However, unlike clinical specimens, the cell lines did not show P-FAK expression in the nuclei. Moreover, the injection of cancer cells into the peritoneal cavity of mice also failed to demonstrate P-FAK expression in the nuclei. These results may be related with the function of carrier proteins of FAK such as Hic-5 and Zyxin, which are found only in humans. Taken together, FAK and P-FAK are involved in the carcinogenesis of digestive organs.
\end{abstract}

Correspondence to: Dr Yoshio Naomoto, Department of Gastrointestinal Surgery, Transplant, and Surgical Oncology, Okayama University Graduate School of Medicine, Dentistry and Pharmaceutical Sciences, 2-5-1 Shikatacho, Okayama 700-8558, Japan E-mail: ynaomoto@md.okayama-u.ac.jp

Key words: FAK, p-FAK, colorectal cancer, carcinogenesis

\section{Introduction}

Focal adhesion kinase (FAK) is a $125-\mathrm{kDa}$ protein tyrosine kinase that was first reported on in 1992. It is an important mediator functioning between cells and the extracellular matrix $(1,2)$. It has been reported that FAK is closely related to the integrin-signaling pathway and is involved in the proliferation, differentiation and apoptosis of cells (3-5). If FAK receives signals from integrin, it is activated and undergoes tyrosine phosphorylation $(6,7)$. Tyrosine 397 is an autophosphorylation site for FAK and serves as a very important component for downstream signaling (8-10). Overexpression of FAK has been reported in many tumor tissues, including in the breast, thyroid, ovary, head and neck region and colon (11-16). However, there are few studies about the expression of tyrosine-phosphorylated FAK (P-FAK). Assuming that FAK plays an important role in signaling, it is significant to check for activated FAK.

To date, no report has demonstrated the involvement of FAK in the carcinogenesis of the digestive tract. Therefore, the present study was undertaken to examine colorectal, esophageal, pancreatic and mammary cancers for expression of FAK and P-FAK by immunohistochemistry. In this study, intense expression of FAK in the cytoplasm was noted (which is consistent with previous reports), but interestingly, P-FAK expression was confined to the nuclei which was an unexpected result. Prior to the present study, P-FAK expression in the nuclei had been reported only for laryngeal cancer and no report of such a finding has been published for cancer of any other organ (17). All four of the organs investigated in the present study showed P-FAK expression in the nuclei, suggesting an association between FAK activation and abnormal cell proliferation.

Colorectal cancer studies at the genetic level have advanced to a level higher than that of any other type of cancer. Recent studies unveiled that there are two major pathways for the onset and progression of colorectal cancer. One of the two pathways is based on the 'adenomacarcinoma sequence', by which normal epithelial cells of mucosa develop adenoma (a polyp), which then advances into a malignant colorectal tumor. This pathway is known to be involved in $\sim 80 \%$ of all cases of sporadic colorectal cancer 
(18-20). The present study was undertaken to check for FAK and tyrosine-phosphorylated FAK (P-FAK) in both early and advanced colorectal cancer cases.

Studies on the carcinogenesis of the esophagus are less advanced than those on colorectal carcinogenesis. However, it has been suggested that esophageal dysplasia is the most important precancerous lesion of the esophagus $(21,22)$. To investigate the expression of FAK and P-FAK in esophageal carcinogenesis, we analyzed esophageal dysplasias in a similar way as colorectal adenomas.

Some investigators reported that an overexpression of FAK had been detected in cancer cell lines in comparison to normal cell lines $(4,23)$. To analyze the expression of FAK and P-FAK in tumor tissues in more detail, it is necessary to analyze the expression using multiple cell lines of the same type of cancer. For this reason, the present study analyzed the expression of FAK and P-FAK using two cell lines of colorectal cancer, i.e., RPMI-4788 and HCC-50. The conditions for the suitable cultivation of cancer cell lines differ from those optimal for the proliferation of cancer cells in vivo. In view of this difference, we implanted RPMI-4788 into the peritoneum of mice to create a mouse model of peritoneal seeded cancer. The implanted cells, undergoing proliferation under conditions identical to those in vivo, were checked for FAK and P-FAK expression to clarify how the expression of these molecules in this model would differ from that of clinical specimens or cell lines.

\section{Materials and methods}

Cells. The human colon cancer cell line, HCC-50, was kindly provided by Dr M. Nishiyama (Department of Biochemistry and Biophysics, Research Institute for Radiation Biology and Medicine, Hiroshima University, Japan). RPMI-4788 was provided by the Roswell Park Memorial Institute, Buffalo, NY. They were incubated in monolayer cultures on RPMI-1640 medium supplemented with $10 \%$ fetal calf serum (FCS), $25 \mathrm{mM}$ HEPES, $100 \mathrm{U} / \mathrm{ml}$ penicillin, and $100 \mathrm{mg} / \mathrm{ml}$ streptomycin in a humidified incubator flushed with $5 \% \mathrm{CO}_{2}$.

Tumor samples. Samples of colorectal, esophageal, pancreatic and mammary tumors used in the study were obtained from surgical patients at the Department of Gastrointestinal Surgery, Transplant and Surgical Oncology of Okayama University Hospital between 1998 and 2000. For immunohistochemical staining, colon adenomas resected endoscopically and stored as paraffin-embedded tissue blocks at Chikuba Anorectal Hospital, Okayama, Japan, during the period from 1997 to 1998 were used. The histological diagnosis was evaluated according to the guidelines of the World Health Organization Classification of Tumours (24).

Immunohistochemistry of cell lines and tumor samples. Cells were seeded onto chamber slides at $2 \times 10^{5}$ cells/slide. After preculture for $48 \mathrm{~h}$, cells were washed once with PBS, and then fixed in cold $20 \%$ formaldehyde for $10 \mathrm{~min}$. Tumor samples of FAK and P-FAK staining were performed using formalin-fixed, paraffin-embedded serial sections. Tissue sections (3- $\mu \mathrm{m}$ thick) were mounted on silanized slides, and were deparaffinized and rehydrated. Immunohistochemical staining was performed using a Histofine SAB PO kit (Nichirei, Tokyo, Japan). Endogenous peroxidase was blocked by incubating the fixed cells in $3 \% \mathrm{H}_{2} \mathrm{O}_{2}$ in methanol for $15 \mathrm{~min}$. Each tumor sample underwent antigen retrieval. Antigen retrieval on paraffin sections was performed by heating them for $5 \mathrm{~min}$, three times in a $10-\mathrm{mM}$ citrate buffer solution $(\mathrm{pH}$ 6.0) in a microwave. After blocking non-specific reactivity with normal goat serum for $10 \mathrm{~min}$ at room temperature, the cells were incubated with the primary antibody overnight at $4^{\circ} \mathrm{C}$. Identification of the distribution of the primary antibody (anti-FAK antibody; Santa Cruz Biotechnology, Santa Cruz, CA anti-pY397 FAK antibody; Biosource International, Inc., CA) was achieved by a subsequent application of a biotinylated anti-primary and streptavidinperoxidase. Immunostaining was detected using a DAB/ hydrogen peroxide solution (Histofine DAB substrate kit; Nichirei), and counterstaining with Mayer's hematoxylin. As a negative control, some sections were subjected to normal serum blocking and omission of the primary antibody. Cases were considered positive when more than $10 \%$ of the atypical cells were stained with the antibody.

Animal experiments. Colon cancer cells (RPMI-4788) were administered $\left(5 \times 10^{6}\right.$ cells) into the abdomens of BALB/C nude mice. For the metastasis analysis, three mice injected with colon cancer cells were sacrificed 30 days later. The peritoneal mass that formed in the peritoneum of each mouse was resected and fixed in formalin. It was then made into paraffin sections. During the experiments, the mice were kept in pathogen-free animal facilities at a controlled temperature and humidity, under a 12-h light/dark cycle, and with food and water provided ad libitum. All animals were acclimatized for at least 1 week before the experiments began. The experimental protocol was approved by the Ethics Review Committee for Animal Experimentation of the Okayama University School of Medicine.

Statistical analysis. Statistical evaluation was calculated using SPSS multiplex comparison software (SPSS Inc., Japan).

\section{Results}

Analysis of FAK and P-FAK expression in colorectal, esophageal, pancreatic and mammary cancers. The expression of FAK and P-FAK in colorectal cancer was examined first. Intense expression of FAK in the cytoplasm was noted, which is consistent with previous reports. P-FAK expression was intense not only in the cytoplasm but also in the nuclei (Fig. 1A). Also in esophageal, pancreatic and mammary cancers, intense expressions of FAK in the cytoplasm and P-FAK in the nuclei were noted (Fig. 1B, C and D). Immunostaining was performed in 5 cases from each disease group, and all of these cases showed P-FAK expression in the nuclei.

Analysis of FAK and P-FAK expression in colorectal adenoma and its difference from that in early colorectal cancer. Sixtyfour cases of colon adenoma were divided based on WHO classifications into low-grade dysplasia and high-grade dysplasia. The expression of FAK and P-FAK in these cases 
A

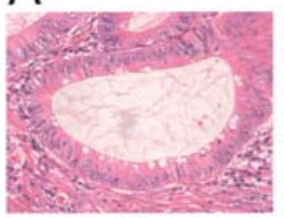

B

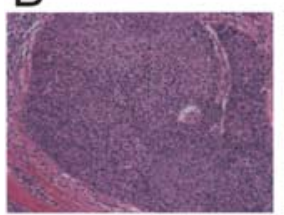

C

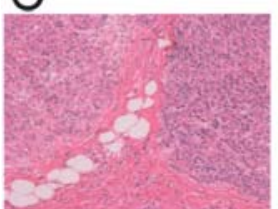

D

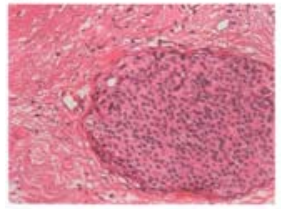

HE
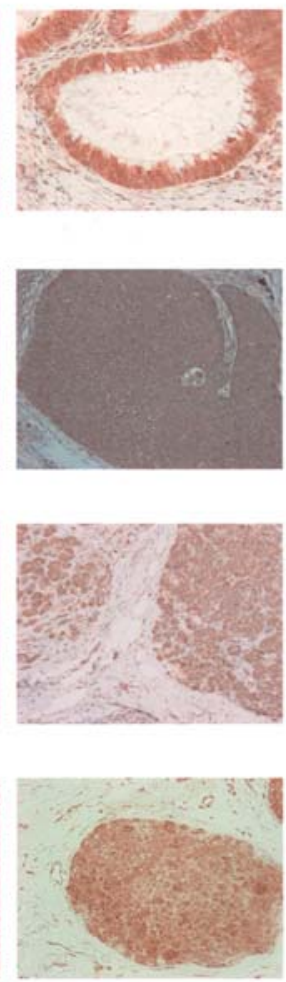

FAK
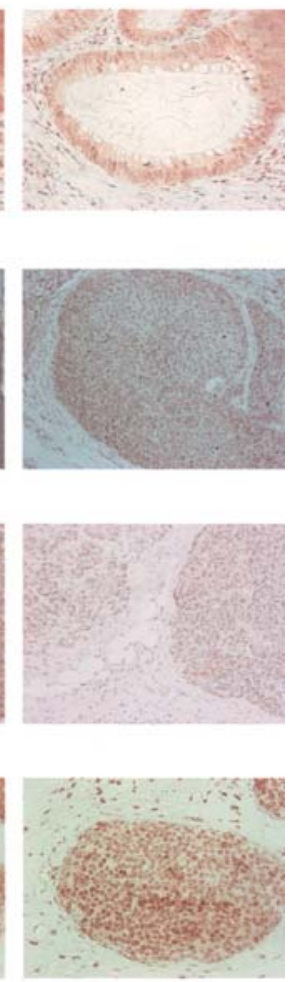

P-FAK

Figure 1. Colorectal (A), esophageal (B), pancreatic (C) and mammary cancers (D) immunostained for FAK and P-FAK. In all cancers, intense FAK expression in the cytoplasm and intense P-FAK expression in the nuclei are noted.

as well as in the intact colorectal mucosa of 35 individuals was analyzed. This analysis involved 36 cases of low-grade dysplasia and 28 cases of high-grade dysplasia. A similar analysis was also conducted on 21 cases of adenocarcinoma with submucosal tumor invasion. In the normal mucosa, the expressions of FAK and P-FAK were weak, but their expression intensified as the degree of mucosal differentiation became higher (Fig. 2). Chromatic responses were scored on a three-point scale (2, positive chromatic responses $\geq 70 \%$ of atypical cells; 1 , positive responses $>10 \%$ and $<70 \%$ of atypical cells; and 0 , positive responses from $\leq 10 \%$ of atypical cells). The scores were compared between the normal group, the low-grade dysplasia group, the high-grade dysplasia group and the adenocarcinoma group. For both FAK and P-FAK expression, the percentage of high-score cases was high in the high-grade dysplasia group and the adenocarcinoma group and low in the low-grade dysplasia group and the normal group (Fig. 3A and C). The expressions of FAK and P-FAK increased significantly in the order of: normal group $<$ low-grade dysplasia group < high-grade dysplasia group $(\mathrm{P}<0.01)$, while there was no significant difference between the high-grade dysplasia group and the adenocarcinoma group (Fig. 3B and D). Also, in the cases of colon adenoma, FAK showed intense expression in the cytoplasm (as seen in cases of colon cancer), but P-FAK expression was intense in the nuclei.

A
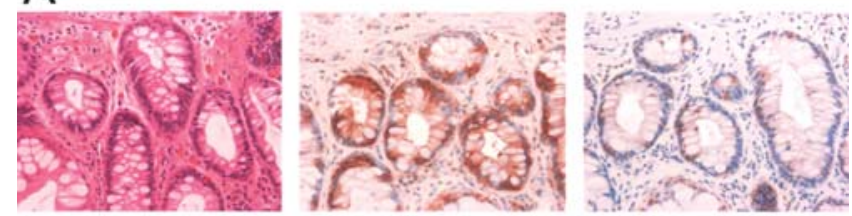

B
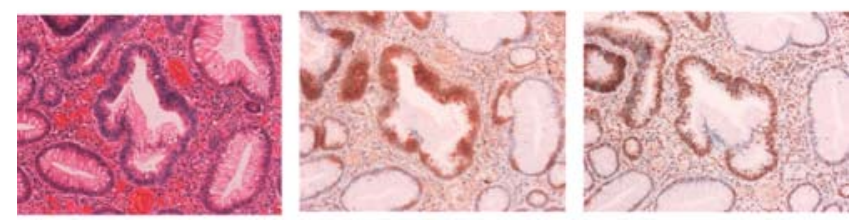

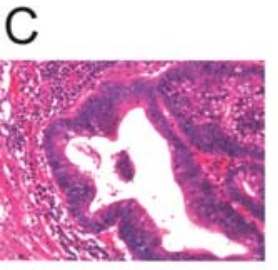

HE

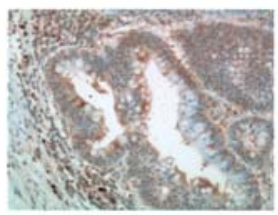

FAK

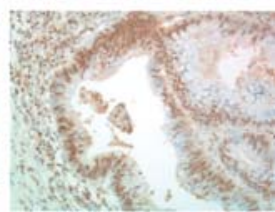

P-FAK
Figure 2. Low-grade dysplasia (A), high-grade dysplasia (B) and adenocarcinoma (tumor invades submucosa) (C) immunostained for FAK and P-FAK. FAK and P-FAK expression is more intense in high-grade dysplasia than in low-grade dysplasia. FAK expression is marked in the cytoplasm, while P-FAK expression is intense in the nuclei.

Analysis of FAK and P-FAK expression in esophageal dysplasia. In cases of esophageal dysplasia, we examined whether or not the expressions of FAK and P-FAK would increase as atypism advanced, like in cases of colon adenoma. Weak expressions of FAK and P-FAK were noted in the normal mucosa and in cases of mild dysplasia lesions, but their expression increased as dysplasia became more severe, which is consistent with the findings from the colon adenoma group (Fig. 4). Also, in cases of esophageal dysplasia, the cytoplasm showed intense FAK expression and the nuclei showed intense P-FAK expression, similar to the cases of colon adenoma.

Analysis of FAK and P-FAK expression in colon cancer cell lines. FAK and P-FAK expressions in two colon cancer cell lines (RPMI-4788 and HCC-50) were analyzed. In both cell lines, the expressions were confined to the cytoplasm, and the nuclei expressed neither FAK nor P-FAK (Fig. 5). When two cell lines of esophageal cancer (TE-1 and T.Tn) were investigated, P-FAK was also not expressed in the nuclei (data not shown).

Analysis of FAK and P-FAK expression in mice with peritoneal seeded tumors. RPMI-4788 cells were injected into the peritoneal cavity of mice to create a model of peritoneal seeding. The peritoneal mass that formed was resected and checked for FAK and P-FAK expression. Although the RPMI-4788 tumor cells were incubated in this model under conditions close to natural conditions in vivo, P-FAK expression in the 
A

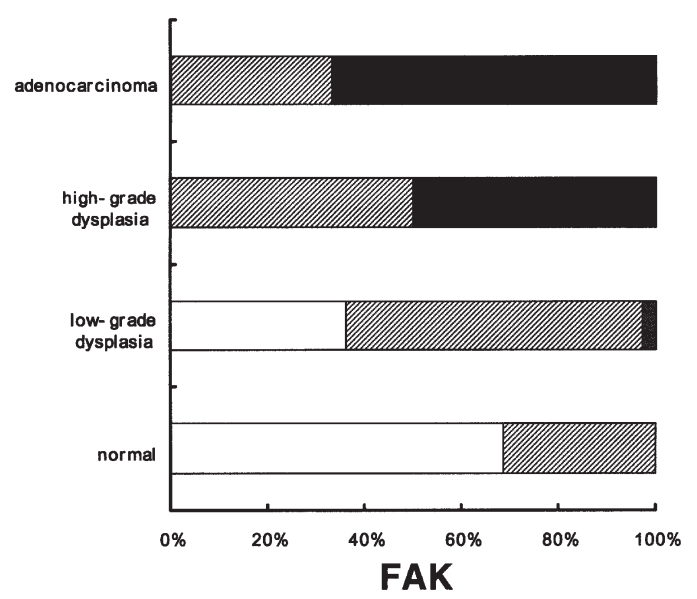

B

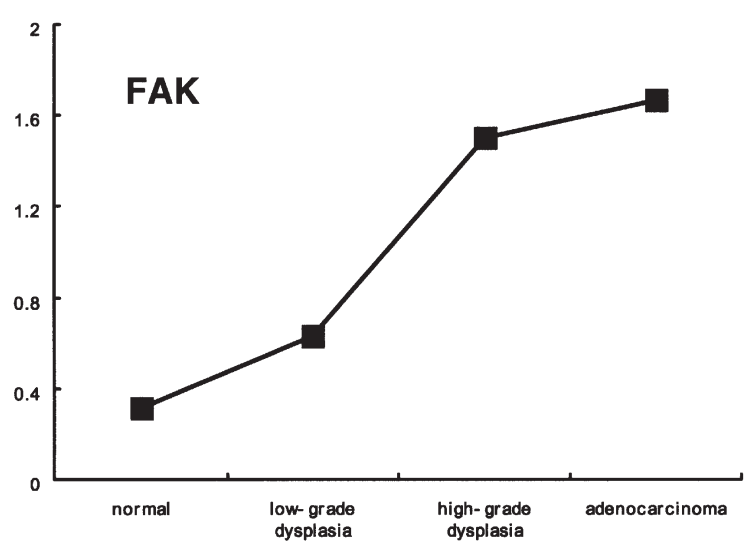

C

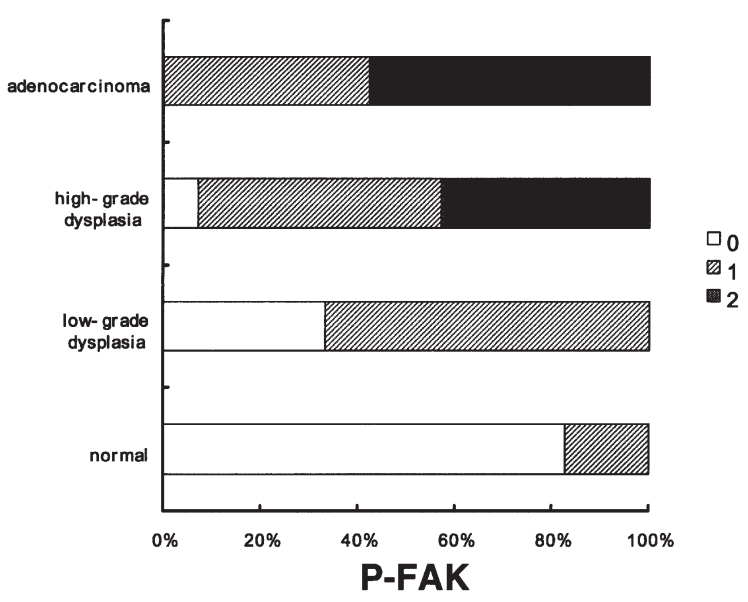

D

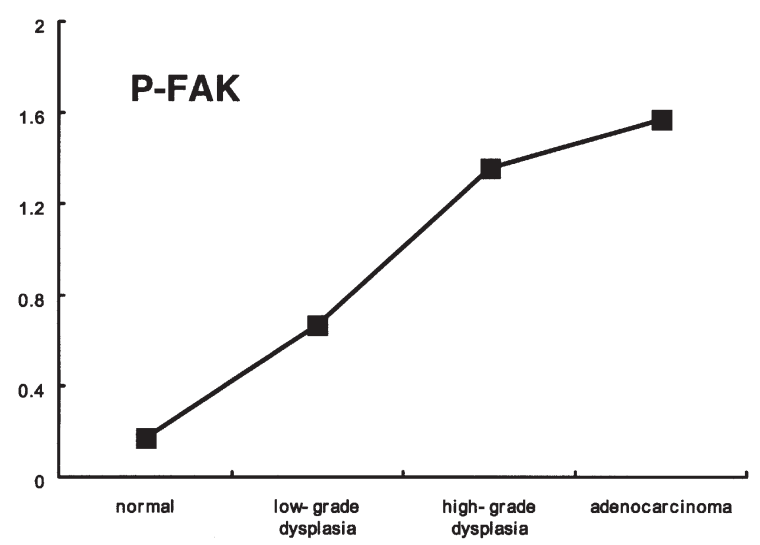

Figure 3. The distribution of FAK and P-FAK in each disease group was scored on a three-point scale (2, intense expression; 1, moderate expression; 0, weak expression). When FAK expression was compared among the different groups, intense expression was often seen in the adenocarcinoma group and seen less frequently in the normal group (A). The mean score differed significantly between each pair of groups $(\mathrm{P}<0.05)$ except for between the high-grade dysplasia group and the adenocarcinoma group (B). When the same analysis was conducted on P-FAK, the expression increased in the order of: normal group < lowgrade dysplasia group < high-grade dysplasia group, but it did not differ significantly between the high-grade dysplasia group and the adenocarcinoma group (C and D).

nuclei was absent, the same as in the experiment involving incubation in vitro (Fig. 6).

\section{Discussion}

FAK is found in the focal adhesion of cells. When a cell binds to the adhesion protein of the extracellular matrix via integrin, FAK undergoes tyrosine phosphorylation, resulting in the secondary induction of various docking proteins and the transduction of signals into the cells $(4,5)$. To date, various studies have been conducted on FAK, but few studies have been carried out on P-FAK. In the present study, we demonstrated that FAK moves into the nuclei following activation in cases of colorectal, esophageal, pancreatic and mammary cancers. Prior to the present study, P-FAK expression in the nuclei had been reported only for laryngeal cancer, and no study had demonstrated the expression of P-FAK in the nuclei of the cells of multiple organs (17). In the present study, immunostaining of P-FAK was performed in cell lines to examine the significance of its expression in the nuclei. However, unlike clinical specimens, the cell lines failed to show P-FAK expression in the nuclei. Graham and Graeme reported that the activation of the FceRI receptor of mast cells resulted in the importation of NH2-terminal FAK into the nuclei (25). It has also been reported that when other cell lines were studied, a cleavage of FAK and its expression in the nuclei were noted (26-30). We also incubated colon cancer cell lines in dishes coated with Matrigel, fibronectin, type-I collagen, etc., and stimulated the cells with EGF, but P-FAK expression in the nuclei was not noted (data not shown). To incubate cells under conditions closer to the environment in vivo, we injected cancer cells into the peritoneal cavity of mice and checked the lesions for P-FAK expression in the nuclei. However, P-FAK expression in the nuclei was not seen in this experiment. Focal adhesion is known to have the proteins Hic-5 and Zyxin, which are involved in intranuclear transfer. These proteins may serve as carriers for intranuclear transfer (31-33). Taken together, the findings from previous and present studies suggest that some particular proteins which are found only in humans serve as the carrier for the transfer of FAK into the nuclei, resulting in P-FAK expression in the nuclei. It is desirable to study such carrier proteins in depth in the future.

The anti-FAK antibody we used is a polyclonal antibody specific to the $\mathrm{NH}_{2}$-terminal. Because the anti-P-FAK antibody recognizes Tyr 397 lesions, cases showing expression 

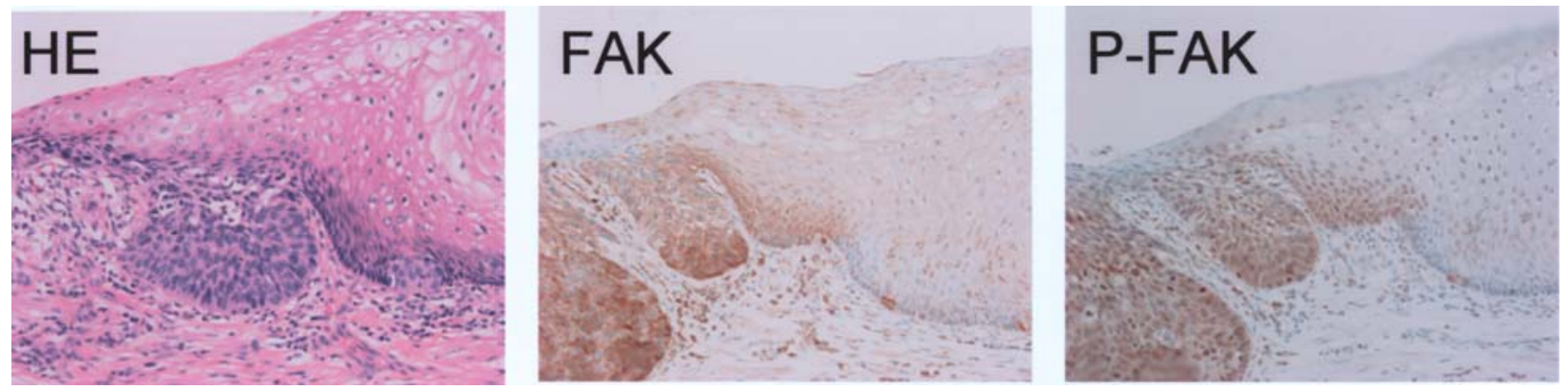

Figure 4. FAK and P-FAK expression in esophageal dysplasia was analyzed. Intense expression of FAK and P-FAK was noted in areas showing high degrees of differentiation, while no expression was seen in normal-appearing areas.
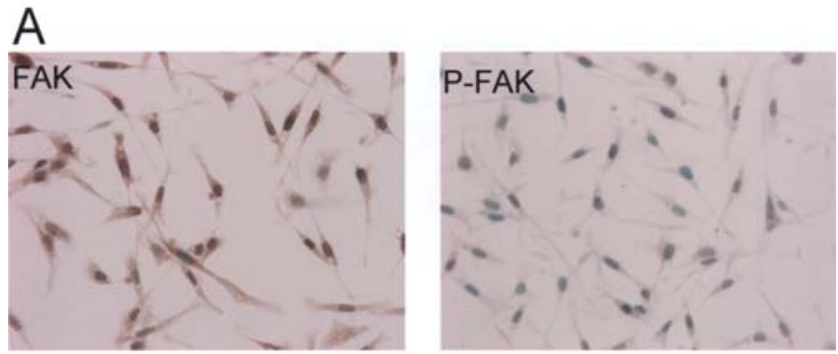

\section{B}
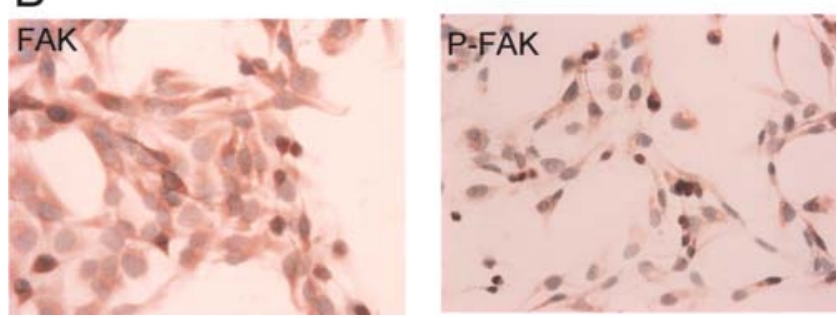

Figure 5. The expression of FAK and P-FAK in two colon cancer cell lines, RPMI-4788 (A) and HCC-50 (B), was analyzed. FAK expression was noted in the cytoplasm, while P-FAK expression in the nuclei was not noted.

of P-FAK in the nuclei should show FAK expression in the nuclei even if FAK has been cleaved to remain in the FERM domain alone $(34,35)$. Following this view, we checked clinical specimens for FAK expression in detail, and found FAK expression in the nuclei of some specimens. Lobo et al reported the detection of $\mathrm{NH}_{2}$-terminal domain fragments of FAK in the nuclei of endothelial cells (27). In the present study it was shown that the $\mathrm{NH}_{2}$-terminal domain of FAK was originally present in the nuclei and that the level of FAK expression rose as the domain was activated.

Thus, in the present study we analyzed the expression of FAK and P-FAK in colon adenoma and demonstrated that their expression increased as the degree of cell differentiation became higher. This finding suggests that FAK and P-FAK play an important role in the process of colorectal carcinogenesis called the 'adenoma-carcinoma sequence'. Similar results were also obtained from esophageal cancer, suggesting that the activation of FAK and P-FAK is involved in the carcinogenesis of the digestive organs. Prior to the present study, no report on the involvement of P-FAK in carcinogenesis has been published. The involvement of FAK in carcinogenesis had been reported only for cervical cancer (16), and the present study first demonstrated that FAK is involved in the carcinogenesis of the digestive tract.

We demonstrated in this study that FAK, which was previously considered to be present in focal adhesion, is present in nuclei, and that its expression increases following activation. Thus, it is essential to study the mechanism for the transfer of activated FAK into the nuclei and the role played by FAK within the nuclei.
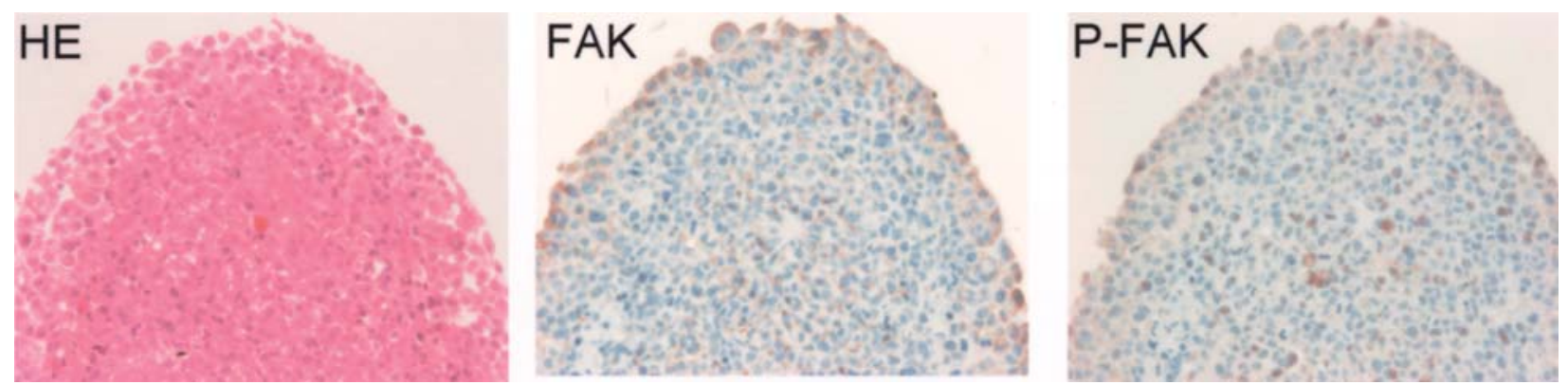

Figure 6. RPMI-4788 was injected into the peritoneal cavity of mice to create a model of peritoneal seeding. The expression of FAK and P-FAK that formed in the peritoneal masses was analyzed. Neither FAK nor P-FAK was expressed. 


\section{References}

1. Hanks SK, Calalb MB, Harper MC and Patel SK: Focal adhesion protein-tyrosine kinase phosphorylated in response to cell attachment to fibronectin. Proc Natl Acad Sci USA 89: 8487-8491, 1992 .

2. Schaller MD, Borgman CA, Cobb BS, Vines RR, Reynolds AB and Persons JT: pp125FAK a structurally distinctive proteintyrosine kinase associated with focal adhesions. Proc Natl Acad Sci USA 89: 5192-5196, 1992.

3. Sonoda Y, Matsumoto Y, Funakoshi M, Yamamoto D, Hanks SK and Kasahara T: Anti apoptotic role of focal adhesion kinase (FAK): J Biol Chem 275: 16309-16315, 2000.

4. Gabarra-Niecko V, Schaller MD and Unty JM: FAK regulates biological processes important for the pathogenesis of cancer. Cancer Metastasis Rev 22: 359-374, 2003.

5. Parsons JT: Focal adhesion kinase: the first ten years. J Cell Sci 116: 1409-1416, 2003.

6. Jones G, Machado J Jr and Tolnay M: PTEN-independent induction of caspase-mediated cell death and reduced invasion by the focal adhesion targeting domain (FAT) in human astrocytic brain tumors which highly express focal adhesion kinase (FAK). Cancer Res 61: 5688-5691, 2001.

7. Schwartz MA and Ginsberg MH: Networks and crosstalk: integrin signaling spreads. Nat Cell Biol 4: E65-E68, 2002.

8. Schlaepfer DD and Mitra SK: Multiple connections link FAK to cell motility and invasion. Cur Opin Genet Dev 14: 92-101, 2004.

9. Matkowskyj KA, Keller K and Glover S: Expression of GRP and its receptor in well-differentiated colon cancer cells correlates with the presence of focal adhesion kinase phosphorylated at tyrosines 397 and 407. J Histochem Cytochem 51: 1041-1048, 2003.

10. Golubovskaya VM, Gross S and Kaur AS: Simultaneous inhibition of focal adhesion kinase and SRC enhances detachment and apoptosis in colon cancer cell lines. Mol Cancer Res 1: 755-764, 2003.

11. Cance WG, Harris JE, Iacocca MV, Roche E, Yang X, Chang J, Simkins S and Xu L: Immunohistochemical analyses of focal adhesion kinase expression in benign and malignant human breast and colon tissues. Clin Cancer Res 6: 2417-2423, 2000.

12. Nix DA and Beckerle MC: Nuclear-cytoplasmic shuttling of the focal contact protein, zyxin: a potential mechanism for communication between sites of cell adhesion and the nucleus. J Cell Biol 138: 1139-1147, 1997.

13. Judson PL, He X, Cance WG and van Le L: Overexpression of focal adhesion kinase, aprotein tyrosine kinase, in ovarian carcinoma. Cancer 86: 1551-1556, 1999.

14. Kornberg LJ: Focal adhesion kianse and its potential involvement in tumor invasion and metastasis. Head Neck 20: 745-752, 1998.

15. Lark AL, Livasy CA and Calvo B: Overexpression of focal adhesion kinase in primary colorectal carcinomas and colorectal liver metastases: immunohistochemistry and real-time PCR analyses. Clin Cancer Res 9: 215-222, 2003.

16. Moon HS, Park WI, Choi EA, Chung HW and Kim SC: The expression and tyrosine phosphorylation of E-cadherin/catenin adhesion complex and focal adhesion kinase in invasive cervical carcinomas. Int J Gynecol Cancer 13: 640-646, 2003.

17. Aronsohn MS, Brown HM and Hauptman G: Expression of focal adhesion kinase and phosphorylated focal adhesion kinase in squamous cell carcinoma of the larynx. Laryngoscope 113: 1944-1948, 2003.
18. Day DW and Morson BC: The adenoma-carcinoma sequence. Major Probl Pathol 10: 58-71, 1978.

19. Hill MJ, Morson BC and Bussey HJ: Aetiology of adenomacarcinoma sequence in large bowel. Lancet 4: 245-247, 1978.

20. Sutherland JV and Bailar JC III: The multihit model of carcinogenesis: etiologic implications for colon cancer. J Chronic Dis 37: 465-480, 1984.

21. Sugimachi K, Sumiyoshi K and Nozoe T: Carcinogenesis and histogenesis of esophageal carcinoma. Cancer 75: 1440-1445, 1995.

22. Lehrbach DM, Nita ME and Cecconello I: Molecular aspects of esophageal squamous cell carcinoma carcinogenesis. Arq Gastroenterol 40: 256-261, 2004.

23. Agochiya M, Brunton VG and Owens DW: Increased dosage and amplification of the focal adhesion kinase gene in human cancer cells. Oncogene 18: 5646-5653, 1999.

24. Hamilton SR and Aaltonen LA: Pathology and Genetics of Tumours of the Digestive System. World Health Organization Classification of Tumours. IARC Press, Lyon, 2000.

25. Graham J and Graeme S: Nuclear import of N-terminal FAK by activation of FceRI receptor in RBL-2H3 cells. Biochem Biophys Res Commun 314: 39-45, 2004.

26. Graham J, Joel MJ and Adrian M: Loss of focal adhesion jinase (FAK) inhibits epidermal growth factor receptor-dependent migration and induces aggregation of N-terminal FAK in the nuclei of apoptotic glioblastoma cells. Cancer Res 61: 4978-4981, 2001.

27. Mel L and Ian Z: Nuclear localization and apoptotic regulation of an amino-terminal domain focal adhesion kinase fragment in endothelial cells. Biochem Biophys Res Commun 276: 1068-1074, 2000.

28. Alasdair S, Claire $\mathrm{H}$ and Ian $\mathrm{Z}$ : The focal adhesion kinase amino-terminal domain localises to nuclei and intercellular junctions in HEK 293 and MDCK cells independently of tyrosine 397 and the carboxy-terminal domain. Biochem Biophys Res Commun 299: 62-73, 2002.

29. Kadare G, Toutant M and Formstecher E: PIAS1-mediated sumoylation of focal adhesion kinase activates its autophosphorylation. J Biol Chem 278: 47434-47440, 2003.

30. Carragher NO, Levkau B and Ross R: Degraded collagen fragments promote rapid disassembly of smooth muscle focal adhesions that correlates with cleavage of pp125(FAK), paxillin and talin. J Cell Biol 147: 619-630, 1999.

31. Su JM, Gui L, Zhou YP and Zha XL: Expression of focal adhesion kinase and alpha5 and beta 1 integrins in carcinomas and its clinical significance. World J Gastroenterol 8: 613-618, 2002.

32. Owens LV, Xu L, Dent GA, Yang X, Sturge GC, Carven RJ and Cance WG: Focal adhesion kinase as a marker of invasive potential in differentiated human thyroid cancer. Ann Surg Oncol 3: 100-105, 1996.

33. Aoto H, Sasaki H and Ishino M: Nuclear translocation of cell adhesion kinase beta/proline-rich tyrosine kinase 2. Cell Struct Funct 27: 47-61, 2002.

34. Girault JA, Labesse G and Mornon JP: The N-termini of FAK and JAKs contain divergent band 4.1 domains. Trends Biochem Sci 24: 54-57, 1999.

35. Sun CX, Robb VA and Gutmann DH: Protein 4.1 tumor suppressors: getting a FERM grip on growth regulation. J Cell Sci 115: 3991-4000, 2000. 